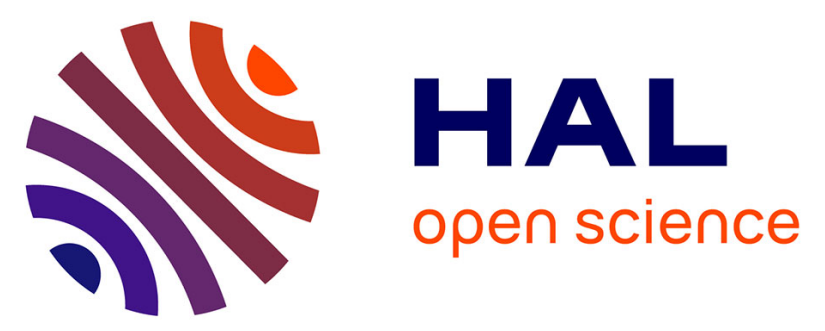

\title{
Nonlinear parabolic equation model for finite-amplitude sound propagation in an inhomogeneous medium over a non-flat, finite-impedance ground surface
} T. Leissing, P. Jean, J. Defrance, Christian Soize

\section{- To cite this version:}

T. Leissing, P. Jean, J. Defrance, Christian Soize. Nonlinear parabolic equation model for finiteamplitude sound propagation in an inhomogeneous medium over a non-flat, finite-impedance ground surface. Acoustics'08, Second ASA (Acoustical Society of America) - EAA (European Acoustics Association) joint international conference, Jun 2008, Paris, France. pp.1-6. hal-00691718

\author{
HAL Id: hal-00691718 \\ https://hal.science/hal-00691718
}

Submitted on 26 Apr 2012

HAL is a multi-disciplinary open access archive for the deposit and dissemination of scientific research documents, whether they are published or not. The documents may come from teaching and research institutions in France or abroad, or from public or private research centers.
L'archive ouverte pluridisciplinaire HAL, est destinée au dépôt et à la diffusion de documents scientifiques de niveau recherche, publiés ou non, émanant des établissements d'enseignement et de recherche français ou étrangers, des laboratoires publics ou privés. 


\title{
ACOUSTICS2008/1961 \\ Nonlinear parabolic equation model for finite-amplitude sound propagation in an inhomogeneous medium over a non-flat, finite-impedance ground surface
}

\author{
T. Leissing ${ }^{\mathrm{a}}$, P. A H Jean ${ }^{\mathrm{a}}$, J. Defrance ${ }^{\mathrm{a}}$ and C. Soize ${ }^{\mathrm{b}}$

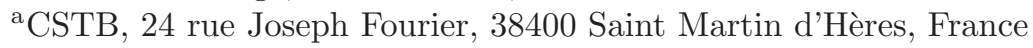 \\ bUniversité Paris-Est, Laboratoire de Mecanique, 5 bd Descartes, 77454 Marne-la-Vallee, France \\ thomas.leissing@cstb.fr
}

\begin{abstract}
A nonlinear parabolic equation (NPE) model for weakly nonlinear sound propagation in an inhomogeneous medium is described. The model being formulated in the time domain, complex impedances cannot be used to simulate ground surfaces. A second NPE model is thus derived to include the medium in the computational system. Based on a nonlinear extension of the Zwikker-Kosten model for rigidly-framed porous media, it allows to include Forchheimer's nonlinearities. Both models are then adapted to terrain-following coordinates, and used together with an interface condition, allow to simulate finite-amplitude sound propagation over a nonflat, finite-impedance ground surface. Numerical examples show that the NPE model is in good agreement with the solutions of the frequency domain boundary element method. Applications of this model to the simulation of sound propagation from explosions in air are then discussed.
\end{abstract}

Number of words in abstract: 133

Keywords: nonlinear acoustics, explosion, shock, outdoor sound propagation, FDTD

Technical area: Noise (NS) - Euronoise

Session: NS12 - Sound and vibration from explosions in air

PACS \#1: 43.25.Cb Macrosonic propagation, finite amplitude sound; shock waves (see also 43.28.Mw and 43.30.Lz)

PACS \#2: 43.28.En Interaction of sound with ground surfaces, ground cover and topography, acoustic impedance of outdoor surfaces

PACS \#3: 43.28.Js Numerical models for outdoor propagation

Presentation: Oral presentation preferred

Special facility: No special equipment

Best student paper competition: yes

Send notice to: Leissing Thomas (thomas.leissing@cstb.fr) 
A nonlinear parabolic equation (NPE) model for weakly nonlinear sound propagation in an inhomogeneous medium is described. The model being formulated in the time domain, complex impedances cannot be used to simulate ground surfaces. A second NPE model is thus derived to include the medium in the computational system. Based on a nonlinear extension of the Zwikker-Kosten model for rigidly-framed porous media, it allows to include Forchheimer's nonlinearities. Both models are then adapted to terrain-following coordinates, and used together with an interface condition, allow to simulate finite-amplitude sound propagation over a non-flat, finite-impedance ground surface. Numerical examples show that the NPE model is in good agreement with the solutions of the frequency domain boundary element method. Applications of this model to the simulation of sound propagation from explosions in air are then discussed.

\section{Introduction}

Due to their high amplitudes, sound waves from explosions propagate over large distances. The need to develop numerical models that can handle main features of finite-amplitude sound propagation outdoors is obvious. Specifically, in addition to nonlinearities, numerical models must take into account meteorological and ground effects (refraction, dissipation, hilly terrain, ground impedance).

Nonlinear parabolic equation (NPE) models can be used to propagate weak shocks over moderate distance. Atmospheric refraction and dissipation can be taken into account. In this work, a NPE model that includes ground effects (topography and impedance) is proposed. The ground layer, characterized by a Zwikker-Kosten (ZK) model, is included as a propagation medium in the calculations. The derivation of the NPE model for porous ground layers is described in section 2. Combined with a boundary interface condition, presented in section 3, and a NPE model for atmospheric media, it allows to simulate finite-amplitude sound propagation over an impedant ground surface. Section 4 aims at adapting the previously developed equations to handle non-flat topographies. Terrain-following coordinates formulations are used. Finally, the use of this model for simulation of waves from explosions in air is discussed (section 6).

\section{NPE model for rigidly-framed porous media}

The domain considered is two-dimensional with main axes $x$ (horizontal direction) and $z$ (vertical direction). The problem is azimuthally symmetric. Total density $\rho_{T}$ and total pressure $p_{T}$ variables are noted as follows:

$$
\rho_{T}=\rho_{0}+\rho^{\prime} \quad p_{T}=p_{0}+p^{\prime}
$$

where $\rho_{0}$ and $p_{0}$ are ambient density and ambient pressure, respectively, and $\rho^{\prime}$ and $p^{\prime}$ are acoustic perturbations of these quantities. Components of the flow vector $\mathbf{V}$ are $u$ and $w$, which are the flow velocities in the $x$ and $z$-directions, respectively. Partial derivation with respect to the variable $i$ is noted $\partial_{i}$.

The nonlinear parabolic equation (NPE) model for sound propagation in porous ground media is based on a nonlinear extension of the Zwikker-Kosten (ZK) model [1]. The ground is considered equivalent to a continuous fluid for sound waves. A sound wave causes a vibration of air particles contained in the ground pores, while the ground frame does not vibrate. The ground layer is characterized by a set of 4 parameters: the DC flow resistivity $\sigma_{0}$, the porosity $\Omega_{0}$, the tortuosity $\Phi$ and the Forchheimer's nonlinearity parameter $\xi$. These quantities are assumed fixed in space and time. In this context, equations of continuity and conservation of momentum are $[2,3,4]$ :

$$
\begin{gathered}
\partial_{t} \rho_{T}+\partial_{x}\left(\rho_{T} u\right)+\partial_{z}\left(\rho_{T} w\right)=0 \\
\Phi \partial_{t}\left(\rho_{T} u\right)+\partial_{x}\left(p_{T}+\Phi \rho_{T} u^{2}\right)+\partial_{z}\left(\Phi \rho_{T} u w\right) \\
+\sigma_{0} \Omega_{0}(1+\xi|u|) u=0 \\
\Phi \partial_{t}\left(\rho_{T} w\right)+\partial_{z}\left(p_{T}+\Phi \rho_{T} w^{2}\right)+\partial_{x}\left(\Phi \rho_{T} u w\right) \\
+\sigma_{0} \Omega_{0}(1+\xi|w|) w=0
\end{gathered}
$$

Combining Eqs (2) and eliminating terms of third order in $x$-derivatives and of second order in $z$-derivatives gives:

$$
\begin{aligned}
\Phi \partial_{t}^{2} \rho_{T} & =\partial_{x}^{2}\left(p_{T}+\Phi \rho_{0} u^{2}\right)+\partial_{z}^{2} p_{T} \\
& +\sigma_{0} \Omega_{0} \partial_{x}[(1+\xi|u|) u]+\sigma_{0} \Omega_{0} \partial_{z} w
\end{aligned}
$$

To find an expresion for the flow velocities $u$ and $w$ we use the perturbation expensions method. The same scalings and expansions as in refs $[5,6]$ are used ${ }^{1}$. Eq (2a) can be rewritten:

$$
\begin{aligned}
& \left(\epsilon \partial_{t}-\frac{c_{0}}{\sqrt{\Phi}} \partial_{x}\right)\left(\rho_{0}+\epsilon \rho_{1}^{\prime}+\epsilon^{2} \rho_{2}^{\prime}+\cdots\right)= \\
& -\partial_{x}\left[\left(\rho_{0}+\epsilon \rho_{1}+\epsilon^{2} \rho_{2}+\cdots\right)\left(\epsilon u_{1}+\epsilon^{3 / 2} u_{2}+\cdots\right)\right] \\
& -\epsilon^{1 / 2} \partial_{z}\left[\left(\rho_{0}+\epsilon \rho_{1}+\epsilon^{2} \rho_{2}+\cdots\right)\left(\epsilon w_{1}+\epsilon^{3 / 2} w_{2}+\cdots\right)\right]
\end{aligned}
$$

Equating terms of order $\epsilon$ and $\epsilon^{3 / 2}$ gives:

$$
u_{1}=\frac{c_{0}}{\sqrt{\Phi}} \frac{\rho_{1}}{\rho_{0}} \quad ; \quad w_{1}=0
$$

Note that $\rho^{\prime}=\rho_{1}+O\left(\epsilon^{2}\right), u=u_{1}+O\left(\epsilon^{3 / 2}\right)$ and $w=w_{1}+O\left(\epsilon^{3 / 2}\right)$. Substitution of $u$ and $w$ by $u_{1}$ and $w_{1}$ in Eq (3) leads to an error consistent with the accuracy sought. The total pressure $p_{T}$ is substituted by a second-order expansion in $\rho^{\prime}$ from an assumed adiabatic equation of state:

$$
p_{T}=p_{0}+c_{0}^{2} \rho^{\prime}+c_{0}^{2}\left(\frac{\gamma-1}{2 \rho_{0}}\right) \rho^{\prime 2}
$$

\footnotetext{
${ }^{1}$ However, note that the sound speed in the ground layer is $c_{0} / \sqrt{\Phi}$.
} 
where $\gamma$ is the ratio of specific heats at constant pressure and volume. Inserting Eq (6) in Eq (3) yields:

$$
\begin{aligned}
\Phi \partial_{t}^{2} \rho^{\prime} & =c_{0}^{2} \partial_{x}^{2}\left[\rho^{\prime}+\left(\frac{\gamma+1}{2 \rho_{0}}\right) \rho^{\prime 2}\right]+c_{0}^{2} \partial_{z}^{2} \rho^{\prime} \\
& +\frac{\sigma_{0} \Omega_{0} c_{0}}{\rho_{0} \sqrt{\Phi}} \partial_{x}\left[\left(1+\frac{\xi c_{0}}{\sqrt{\Phi}}\left|\frac{\rho^{\prime}}{\rho_{0}}\right|\right) \rho^{\prime}\right]
\end{aligned}
$$

A "moving-frame" operator $D_{t}^{\star}$ is introduced:

$$
D_{t}^{\star}=\partial_{t}+\frac{c_{0}}{\sqrt{\Phi}} \partial_{x}
$$

The parabolic approximation gives [7]:

$$
\partial_{t}^{2} \longrightarrow-2 \frac{c_{0}}{\sqrt{\Phi}} D_{t}^{\star} \partial_{x}+\frac{c_{0}^{2}}{\Phi} \partial_{x}^{2}
$$

Replacing the second time derivative in Eq (7) and rearranging gives a NPE model for propagation in porous media:

$$
\begin{aligned}
D_{t}^{\star} R & =-\frac{c_{0}}{\sqrt{\Phi}} \partial_{x}\left(\frac{\beta}{2} R^{2}\right)-\frac{c_{0}}{2 \sqrt{\Phi}} \int \partial_{z}^{2} R d x \\
& -\frac{\sigma \Omega}{2 \Phi \rho_{0}}\left(1+\frac{\xi c_{0}}{\sqrt{\Phi}}|R|\right) R
\end{aligned}
$$

where $\beta$ is the hydrodynamic nonlinearity parameter and $R$ is a dimensionless density perturbation $(R=$ $\left.\rho^{\prime} / \rho_{0}\right)$. Eq (10) can be used to simulate sound propagation in a porous ground. Note that if $\Phi=1$ and losses in the layer are neglected, the model exactly reduces to the usual NPE model for atmospheric propagation [8]. However, if one wants to couple air/ground models, a last modification must be done. Indeed, both models use different frame speeds: $c_{0}$ and $c_{0} / \sqrt{\Phi}$. Correcting for the frame-speed difference leads to the following substitution:

$$
D_{t}^{\star} \longrightarrow D_{t}+\frac{c_{0}}{\sqrt{\Phi}}(1-\sqrt{\Phi}) \partial_{x}
$$

\section{Derivation of a boundary inter- face condition}

As both models use the same moving-frame speed, they can be combined to simulate finite-amplitude sound propagation over a rigidly-framed porous ground layer. This section aims at establishing a first-order boundary interface condition to link these two propagation models. The variables $p_{i, j}^{\prime a}$ and $p_{i, j}^{\prime g}$ are introduced to denote quantities in layer $A$ (air layer) and layer $G$ (porous ground layer), respectively, at range $i \Delta x$ in the moving window and altitude $j \Delta z$. The fluid-fluid interface is taken to be midway between two vertical grid points with indexes $j=0$ and $j=1$. Auxiliary virtual points $p_{i, 0}^{\prime a}$ and $p_{i, 1}^{\prime b}$ are created. In the following we assume that the deformation of the interface by the wave is small [9]. Interfacial boundary conditions are continuity of pressure and normal flow velocity:

$$
\left[p^{\prime a}\right]=\left[p^{\prime g}\right] \quad\left[w^{a}\right]=\left[w^{g}\right]
$$

where the square brackets denote a quantity across the interface. We seek for expressions of $w^{a}$ and $w^{g}$ involving the pressure disturbance $p^{\prime}$ to the first order. As a first order boundary interface condition is sought, linearized equations are used; for the air layer $A$ we use the linearized Euler equation:

$$
\rho_{0} \partial_{t}\left(w^{a}\right)=-\partial_{z} p_{T}^{a}
$$

The same scalings and expansions as in section 2 and in refs $[5,6]$ are used. Rewriting Eq (13) and equating terms of order 1 and $3 / 2$ gives:

$$
w_{1}^{a}=0 \quad w_{2}^{a}=\left(\rho_{0} c_{w} \partial_{x}\right)^{-1} \partial_{z} p_{1}^{\prime a}
$$

Note that $w^{a}=w_{1}^{a}+w_{2}^{a}+O\left(\epsilon^{5 / 2}\right)$. To the order of accuracy sought in this work it can be written:

$$
w^{a}=\left(\rho_{0} c_{0} \partial_{x}\right)^{-1} \partial_{z} p_{1}^{\prime a}
$$

To find an expression for $w^{g}$ we start from the following equation [10]:

$$
\Phi \rho_{0} \partial_{t} w^{g}=-\Omega_{0} \partial_{z} p_{T}^{g}-\sigma_{0} \Omega_{0} w^{g}
$$

The same procedure is applied; one can find:

$$
w^{g}=\left(\sqrt{\Phi} \rho_{0} c_{0} \partial_{x}-\sigma_{0} \Omega_{0}\right)^{-1} \Omega_{0} \partial_{z} p_{1}^{\prime g}
$$

The interfacial condition for the continuity of vertical velocities $w^{a}$ and $w^{g}$ can now be written:

$$
\begin{aligned}
& {\left[\left(\rho_{0} c_{0} \partial_{x}\right)^{-1} \partial_{z} p^{\prime a}\right]=} \\
& \quad\left[\left(\sqrt{\Phi} \rho_{0} c_{0} \partial_{x}-\sigma_{0} \Omega_{0}\right)^{-1} \Omega_{0} \partial_{z} p^{\prime g}\right]
\end{aligned}
$$

Rearranging Eq (18) leads to:

$$
\left[\sqrt{\Phi} \partial_{z} p^{\prime a}-\frac{\sigma_{0} \Omega_{0}}{\rho_{0} c_{0}} \int \partial_{z} p^{\prime a} d x\right]=\left[\Omega_{0} \partial_{z} p^{\prime g}\right]
$$

A trapezoïdal law and finite-differences expressions for $p^{\prime a}$ and $p^{\prime g}$ and their derivatives are used to discretize Eq (19). For a layer $l$ we use:

$$
\left[p^{\prime} l\right]=\frac{p_{i, 1}^{\prime l}+p_{i, 0}^{\prime l}}{2} \quad ;\left[\partial_{z} p^{\prime} l\right]=\left(p_{i, 1}^{\prime l}-p_{i, 0}^{\prime l}\right) \Delta z^{-1}
$$

Replacing these approximations into Eq (19) gives expressions for unknown quantities $p_{i, 0}^{\prime a}$ and $p_{i, 1}^{\prime g}$ :

$$
\begin{aligned}
p_{i, 0}^{\prime a} & =\left(\frac{A_{1}-G_{1}}{A_{0}+G_{1}}\right) p_{i, 1}^{\prime a}+\left(\frac{G_{0}+G_{1}}{A_{0}+G_{1}}\right) p_{i, 0}^{\prime g} \\
& +\left(\frac{S_{A}}{A_{0}+G_{1}}\right) \sum_{m=N_{x}}^{i+1}\left(p_{m, 1}^{\prime a}-p_{m, 0}^{\prime a}\right) \\
p_{i, 1}^{\prime g} & =\left(\frac{G_{0}-A_{0}}{A_{0}+G_{1}}\right) p_{i, 0}^{\prime g}+\left(\frac{A_{0}+A_{1}}{A_{0}+G_{1}}\right) p_{i, 1}^{\prime a} \\
& +\left(\frac{S_{A}}{A_{0}+G_{1}}\right) \sum_{m=N_{x}}^{i+1}\left(p_{m, 1}^{\prime a}-p_{m, 0}^{\prime a}\right)
\end{aligned}
$$

with:

$$
\begin{aligned}
A_{0} & =A_{1}=\sqrt{\Phi}+\frac{\sigma_{0} \Omega_{0} \Delta x}{2 c_{0} \rho_{0}} \\
G_{0} & =G_{1}=\Omega_{0} \\
S_{A} & =\frac{\sigma_{0} \Omega_{0} \Delta x}{c_{0} \rho_{0}}
\end{aligned}
$$


Eqs (21) give expressions for the unknown virtual points $p_{i, 0}^{a}$ and $p_{i, 1}^{g}$, and thus allow, used together with the atmospheric and porous ground NPE models, to simulate weakly nonlinear sound propagation over an impedant ground. If one sets $\Phi=+\infty$ we obtain from Eqs (21): $p_{i, 0}^{\prime a}=p_{i, 1}^{\prime a}$ which is the condition for a hard wall. A transparent interface condition can be obtained by setting $\sigma_{0}=0, \Omega_{0}=1$ and $\Phi=1$ (parameters for an air layer). This leads to: $A_{0}=A_{1}=1$ and $G_{0}=G_{1}=1$ and thus $p_{i, 0}^{\prime a}=p_{i, 0}^{\prime g}$ and $p_{i, 1}^{\prime g}=p_{i, 1}^{\prime a}$. If one sets $\sigma=0$ and $\Omega_{0}=1$, Eqs (21) become:

$$
\begin{aligned}
& p_{i, 0}^{\prime a}=\frac{\sqrt{\Phi}-1}{\sqrt{\Phi}+1} p_{i, 1}^{\prime a}+\frac{2}{\sqrt{\Phi}+1} p_{i, 0}^{\prime g} \\
& p_{i, 1}^{\prime g}=\frac{1-\sqrt{\Phi}}{\sqrt{\Phi}+1} p_{i, 0}^{\prime g}+\frac{2 \sqrt{\Phi}}{\sqrt{\Phi}+1} p_{i, 1}^{\prime a}
\end{aligned}
$$

which is the interface condition for two fluid layers with densities $\rho_{0}$ and $\sqrt{\Phi} \rho_{0}$ [9]. From a numerical point of view, a common way for solving for diffraction is to use first order finite-differences. This leads to a tridiagonal system of equations that is solved columnwise, from the right to the left of the calculation grid. The boundary interface condition can thus be naturally included in the diffraction solver by imposing values on corresponding points without any additional solver modifications.

\section{Propagation over non-flat sur- faces}

For long-range sound propagation applications the need to include the effect of hilly terrain in the model is obvious. To achieve this goal, a convenient method is the use of terrain-following coordinates [11]. The ground height is noted $h(x)$ and its first and second derivatives with respect to $x$ are noted $h^{\prime}$ and $h^{\prime \prime}$, respectively. We use the following transformation :

$$
x \longrightarrow x \quad z \longrightarrow z+h(x)
$$

Using such a transformation has the advantage of conserving spatial resolution over the calculation grid [11].

\subsection{Atmospheric model}

We start from a nonlinear wave equation written in dimensionless form, where nonlinearities have been neglected in the $z$-direction:

$$
\partial_{t}^{2} R=\partial_{x}^{2}\left(c_{0}^{2} R+c_{0}^{2} \beta R^{2}\right)+c_{0}^{2} \partial_{z}^{2} R
$$

In the transformed coordinates system the above equation is:

$$
\partial_{t}^{2} R=\mathfrak{D}_{x}^{2}\left(c_{0}^{2} R+c_{0}^{2} \beta R^{2}\right)+c_{0}^{2} \partial_{z}^{2} R
$$

where $\mathfrak{D}_{x}^{2}$ is the transformed second $x$-derivative:

$$
\begin{aligned}
\mathfrak{D}_{x}^{2} & =\partial_{x}^{2}+h^{\prime 2} \partial_{z}^{2}-h^{\prime \prime} \partial_{z}-2 h^{\prime} \partial_{x} \partial_{z} \\
& =\partial_{x}^{2}+\mathfrak{L}
\end{aligned}
$$

with $\mathfrak{L}=h^{\prime 2} \partial_{z}^{2}-h^{\prime \prime} \partial_{z}-2 h^{\prime} \partial_{x} \partial_{z}$. Using a moving-frame operator (see section 2) leads to:

$$
\begin{aligned}
D_{t} R= & -\partial_{x}\left(c_{0} \frac{\beta}{2} R^{2}\right)-\frac{c_{0}}{2} \int \partial_{z}^{2} R d x \\
& -\int \mathfrak{L}\left[\frac{c_{0}}{2} R+\frac{c_{0} \beta}{2} R^{2}\right] d x
\end{aligned}
$$

Under the assumptions of weak nonlinearities and dominant propagation in the $x$-direction Eq (29) can be reduced to:

$$
\begin{aligned}
D_{t} R= & -\partial_{x}\left[c_{1} R+c_{0} \frac{\beta}{2} R^{2}\right]+c_{0} h^{\prime} \partial_{z} R \\
& -\frac{c_{0}}{2} \int\left[\left(1+h^{\prime 2}\right) \partial_{z}^{2} R+h^{\prime \prime} \partial_{z} R\right] d x
\end{aligned}
$$

The model developed can be used to simulate sound propagation over smooth terrains. However, large slopes may lead to incorrect results: the integral in Eq (29) contains a nonlinear term. Neglecting it implies that ground topography derivatives are small. Note that if the ground elevation $h$ is set to zero, Eq (30), further called Generalized Terrain-NPE (GT-NPE), exactly reduces to the usual NPE.

The NPE model has been proven to be the time-domain counterpart of the frequency domain Parabolic Equation (PE) [5]. The same procedure can be used to prove that the GT-NPE is a proper equivalence of the GT-PE [12]. The derivation is straightforward: dropping the nonlinear term in Eq (30), substituing $R=f(x, z) e^{i(k x-\omega t)}$ and neglecting non-dominant terms gives the first-order "narrow-angle" GT-PE.

\subsection{Porous ground model}

Similarly, a GT-NPE model for porous ground media can be obtained. Using the same assumptions as in section 4.1, transformation of Eq (10) in the terrainfollowing coordinates system is direct. One can obtain:

$$
\begin{aligned}
D_{t} R= & -\frac{c_{0}}{\sqrt{\Phi}} \partial_{x}\left[(1-\sqrt{\Phi}) R+\frac{\beta}{2} R^{2}\right] \\
& -\frac{\sigma \Omega}{2 \Phi \rho_{0}}\left(1+\frac{\xi c_{0}}{\sqrt{\Phi}}|R|\right) R+\frac{c_{0}}{\sqrt{\Phi}} h^{\prime} \partial_{z} R \\
& -\frac{c_{0}}{2 \sqrt{\Phi}} \int\left[\left(1+h^{\prime 2}\right) \partial_{z}^{2} R+h^{\prime \prime} \partial_{z} R\right] d x
\end{aligned}
$$

\subsection{Boundary interface condition}

A boundary interface condition has to be derived for non flat-terrains. The procedure is identical to the one described in section 3. Expressions for the vertical flow velocities are:

$$
\begin{aligned}
w^{a}= & \left(\rho_{0} c_{0} \partial_{x}\right)^{-1}\left[\partial_{z} p_{1}^{\prime a}-h^{\prime} \partial_{x} p_{1}^{\prime}\right] \\
w^{g}= & \left(\sqrt{\Phi} \rho_{0} c_{0} \partial_{x}-\sigma_{0} \Omega_{0}\right)^{-1} \\
& {\left[\Omega_{0} \partial_{z} p_{1}^{\prime g}-h^{\prime}\left(\sqrt{\Phi} \partial_{x}-\frac{\sigma \Omega_{0}}{\rho_{0} c_{0}}\right) p_{1}^{\prime g}\right] }
\end{aligned}
$$



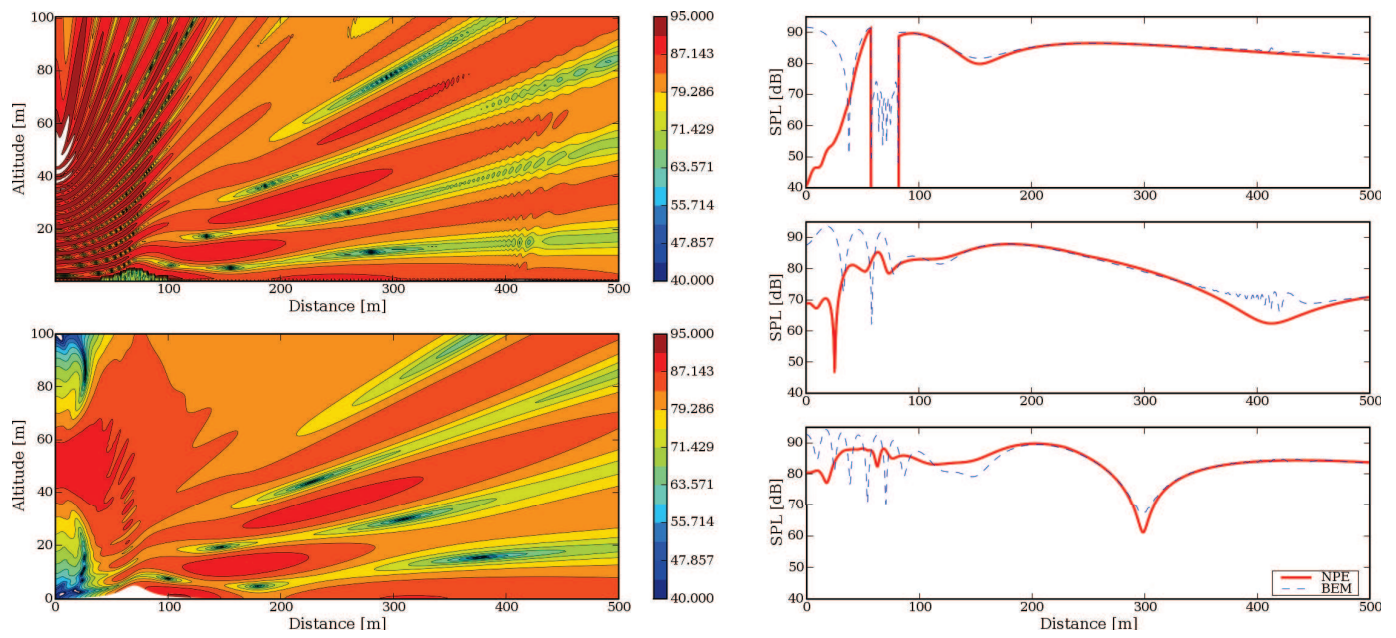

Figure 1: Left: SPL maps for BEM (top) and GT-NPE (bottom) models for sound propagation over a (rigid) hilly ground. Right: SPL slices at altitudes $1.5 \mathrm{~m}$ (top), $15 \mathrm{~m}$ (middle) and $30 \mathrm{~m}$ (bottom).

One can get corrected coefficients to account for elevation in the boundary interface condition:

$$
\begin{aligned}
A_{0}= & \sqrt{\Phi}+\frac{\sigma_{0} \Omega_{0} \Delta x}{2 c_{0} \rho_{0}}\left(1-\frac{h^{\prime \prime} \Delta z}{2}\right) \\
& -h^{\prime} \frac{\Delta z}{2}\left(\frac{\sigma_{0} \Omega_{0}}{\rho_{0} c_{0}}+\frac{\sqrt{\Phi}}{\Delta x}\right) \\
A_{1}= & \sqrt{\Phi}+\frac{\sigma_{0} \Omega_{0} \Delta x}{2 c_{0} \rho_{0}}\left(1+\frac{h^{\prime \prime} \Delta z}{2}\right) \\
& +h^{\prime} \frac{\Delta z}{2}\left(\frac{\sigma_{0} \Omega_{0}}{\rho_{0} c_{0}}+\frac{\sqrt{\Phi}}{\Delta x}\right) \\
G_{0}= & \Omega_{0}-h^{\prime} \frac{\Delta z}{2}\left(\frac{\sigma_{0} \Omega_{0}}{\rho_{0} c_{0}}+\frac{\sqrt{\Phi}}{\Delta x}\right) \\
G_{1}= & \Omega_{0}+h^{\prime} \frac{\Delta z}{2}\left(\frac{\sigma_{0} \Omega_{0}}{\rho_{0} c_{0}}+\frac{\sqrt{\Phi}}{\Delta x}\right)
\end{aligned}
$$

Note that if one set $h=0$, these expressions reduce to the coefficients in section 3 .

\section{Numerical verification}

To verify the correctness of the different NPE models developed a boundary element method code [13] is used to generate reference solutions. Two configurations are considered: propagation over a hilly and rigid ground, and propagation over a flat and impedant ground. Signals amplitudes are low enough for the propagation to be considered linear.

\subsection{Propagation over a hill}

The GT-NPE model is used to study the propagation of a finite-length signal over a hill. The configuration of this example is taken from ref [11]. The ground elevation is given by:

$$
h(x)=h_{\text {top }}\left[1+\frac{\left(x-x_{0}\right)^{2}}{l^{2}}\right]^{-1}
$$

where $h_{\text {top }}$ is the maximum ground elevation, equal to 5 $\mathrm{m}, l$ is the hill width, equal to $15 \mathrm{~m}$ and $x_{0}$ is the maximum elevation location, equal to $70 \mathrm{~m}$. The maximum slope is $\left|h^{\prime}(x)\right|_{\max }=0.22$. The source is positioned at $x=0 \mathrm{~m}, z=50 \mathrm{~m}$ and emits a 8 -period $50 \mathrm{~Hz}$ sine-wave. The sound celerity is constant through the domain and set to $343.4 \mathrm{~m} . \mathrm{s}^{-1}$. Spatial steps are both equal to 30 $\mathrm{cm}$ and the ground layer is 20 point-thick and perfectly rigid.

Figure 1 presents calculation results from both BEM and GT-NPE models. One can see that the parabolic approximation inherant to the GT-NPE model prevents having correct SPLs near the source. At a reasonable distance from the source very good agreement is found between BEM and GT-NPE calculations.

\subsection{Propagation over a ground surface}

The propagation of a broad-band pulse over a flat, impedant ground is studied. The source is positioned at $z=1.4 \mathrm{~m}$ and emits a gaussian pulse with central frequency $f_{c}=850 \mathrm{~Hz}$. A virtual receptor is placed $6 \mathrm{~m}$ away from the source and at altitude $z=1.4$ $\mathrm{m}$. Three different ground layers are considered. The first ground layer is a perfectly rigid surface (large $\Phi$ ). The second and third configuration have identical tortuosity $(\Phi=3)$ and porosity $\left(\Omega_{0}=0.3\right)$, but different flow resistivities $\left(\sigma_{0}=100 \mathrm{kPa.s.m} \mathrm{m}^{-2}\right.$ and $\sigma_{0}=$ 10 kPa.s. $\mathrm{m}^{-2}$ ). The normalized impedance used in the frequency-domain calculations is given by:

$$
Z=\sqrt{\frac{\Phi}{\Omega_{0}^{2}}+i \frac{\sigma_{0}}{\rho_{0} \Omega_{0} \omega}}
$$

Figure 2 shows snapshots of the propagation together with the SPLs relative to free field for the three configurations, for both BEM and NPE calculations. Very good agreement can be observed, even for very soft grounds, where the difference is about $1 \mathrm{~dB}$. 

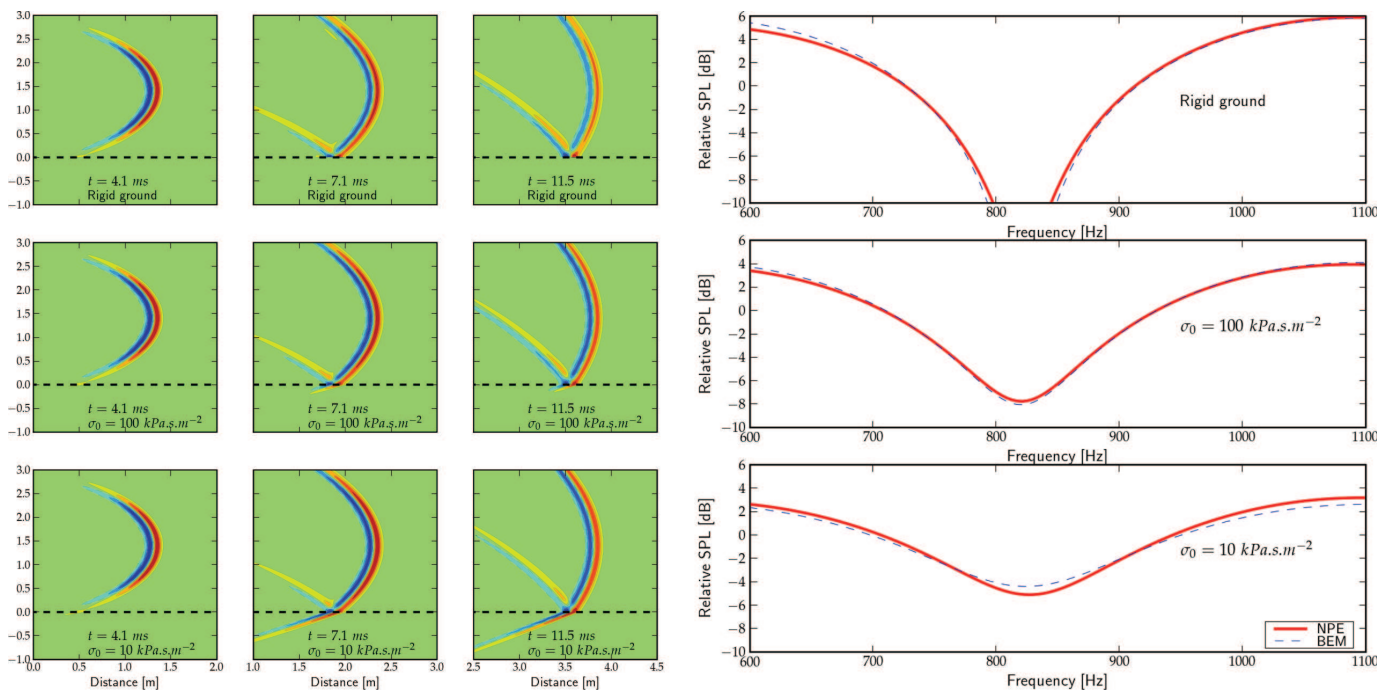

Figure 2: Left: Snapshots at times 4.1, 7.1 and $11.5 \mathrm{~ms}$ of a gaussian pulse propagating over three different ground layers. Right: SPL relative to free field at position $x=6 \mathrm{~m}$ and $z=1.4 \mathrm{~m}$.

\section{Conclusion}

A NPE model for propagation in porous ground layers and a first-order boundary interface condition have been presented. It provides a simple but efficient way of taking into account ground impedances. Propagation over non-flat terrains is handled through the use of terrain-following coordinates, which has been proven to give satisfactory results for gentle slopes. With atmospheric refraction and dissipation included, this work provides a complete NPE model for weakly nonlinear wave propagation. Propagation of waves from explosions can be simulated using a three stages procedure: first, a method based on Euler equations is used in the near field, where the propagation is highly nonlinear. Next, NPE models can propagate weakly nonlinear waves over moderate distances and finally, when the wave amplitude is low enough, (linear) frequencydomain method like the PE can be used. This hybrid method allows to propagate waves from explosions over distances up to several kilometres [14].

\section{References}

[1] C. Zwikker and C. W. Kosten, Sound absorbing materials. Elsevier, 1949.

[2] A. Krylov, S. Sorek, A. Levy, and G. Ben-Dor, "Simple waves in saturated porous media (I. The isothermal case)," JSME international journal, vol. 39, pp. 294-298, 1996.

[3] E. Védy, "Simulations of flows in porous media with a flux corrected transport algorithm," Noise Control Eng. J., vol. 50, pp. 211-217, 2002.

[4] O. Umnova, K. Attenborough, and A. Cummings, "High amplitude pulse propagation and reflection from a rigid porous layer," Noise Control Eng. J., vol. 50, pp. 204-210, 2002.

[5] B. E. McDonald and W. A. Kuperman, "Time domain formulation for pulse propagation including nonlinear behaviour at a caustic," J. Acoust. Soc. of Am., vol. 81, pp. 1406-1417, 1987.

[6] B. E. McDonald, P. Caine, and M. West, "A tutorial on the nonlinear progressive wave equation (NPE) - Part 1," Applied Acoustics, vol. 43, pp. 159-167, 1994.

[7] P. Caine and M. West, "A tutorial on the nonlinear progressive wave equation (NPE) - Part 2. derivation of the three dimensional cartesian version without use of perturbation expansions," $A p$ plied Acoustics, vol. 45, pp. 155-165, 1995.

[8] For example, Eq (31) in [6], or Eq (39) in [7].

[9] J. J. Ambrosiano, D. R. Plante, B. E. McDonald, and W. A. Kuperman, "Nonlinear propagation in an ocean acoustic waveguide," J. Acoust. Soc. of Am., vol. 87, pp. 1473-1481, 1990.

[10] Eq (16) is given as Eq (10) in [15].

[11] R. Karle and D. Heimann, "A linearized eulerian finite difference time domain sound propagation model with terrain following coordinates," $J$. Acoust. Soc. of Am., vol. 119, pp. 3813-3821, 2006.

[12] R. A. Sack and M. West, "A parabolic equation for sound propagation in two dimensions over any smooth terrain profile: the generalized terrain parabolic equation (GT-PE)," Applied Acoustics, vol. 45, pp. 113-129, 1995.

[13] P. Jean, "A variational approach for the study of outdoor sound propagation and application to railway noise," J. Sound Vib., vol. 212, pp. 275-294, 1998.

[14] F. van der Eerden and E. Védy, "Propagation of shock waves from source to receiver," Noise Control Eng. J., vol. 53, pp. 87-93, 2005.

[15] E. M. Salomons, R. Blumrich, and D. Heimann, "Eulerian time-domain model for sound propagation over a finite impedance ground surface. Comparison with frequency-domain models," Acta Acustica United With Acustica, vol. 88, pp. 483492, 2002. 